\title{
CELL TRACKING QUALITY COMPARISON BETWEEN ACTIVE SHAPE MODEL (ASM) AND ACTIVE APPEARANCE MODELS (AAM)- BASED TRACKING
}

\author{
Ashraf A. Aly \\ Department of computer Science and Information Systems, Morehead State University, \\ USA
}

\begin{abstract}
The aim of this paper is to introduce a comparison between cell tracking using active shape model (ASM) and active appearance model (AAM) algorithms, to compare the cells tracking quality between the two methods to track the mobility of the living cells. Where sensitive and accurate cell tracking system is essential to cell motility studies. The active shape model (ASM) and active appearance model (AAM) algorithms has proved to be a successful methods for matching statistical models. The experimental results indicate the ability of (AAM) method to give better accuracy for tracking the mobility of the WBC than (ASM) method.
\end{abstract}

\section{KEYWORDS}

Cell tracking, segmentation enhancement, Active Contour, Topological Alignments.

\section{INTRODUCTION}

Cell tracking and segmentation with high accuracy is important step in the cell motility studies. Understanding the mechanisms of cell motility is essential part for curative and preventative treatments to many diseases. For instance, tracking the number and velocity of rolling leukocytes is essential to understand and successfully treat inflammatory diseases [15]. Sensitive tracking for moving cells is important to do mathematical modeling to cell locomotion. Moreover, Zimmer [17] modified the snake model to track the movement of the cells and segment the first frame. Another research by Mukherjee [19] he developed a technique to handle segmentation process and tracking problem simultaneously. Li [20] used a technique with two stages; the first one is a tracker and a filter to detect the cell and also the cells which move in and out of the image area. Coskun [12] used imaging data to solve the inverse modeling problem to determine the mobility analysis of the cells. Recently, a number of researchers have been created automated techniques to track and detect the cells mobility. Segmentation is an essential part in many signal processing techniques and its applications. Texture analysis is important in many areas such as image processing, determination of the object shape, scene analysis.

The process of segmentation depends on the determination of the best positions of the points which represent the image. The purpose of image segmentation is to partition an image into meaningful regions based on measurements taken from the image and might be grey level, colour,

DOI:10.5121/ijitcs.2016.6402 
texture, depth or motion. Usually the process to determine the image starts with image segmentation as initial step. The goal of image segmentation is to cluster pixels into salient image regions, i.e., regions corresponding to individual objects, surfaces, or natural parts of objects.

Active Shape Models (ASM) was originally designed as a method for locating given shapes or outlines within images (Cootes et al., 2000). An ASM-based procedure starts with the base shape, approximately aligned to the object, iteratively distorts it and refines its pose to obtain a better fit. It seeks to minimize the distance between model points and the corresponding pixels found in the image.

Cootes et al. (2002) introduces the Active Appearance Models (AAM) algorithm. The original algorithm was described by Edwards's et.al. (1998). The Active Appearance Model (AAM) algorithm has been widely used for matching statistical models of appearance to image data. The models, trained on suitably annotated examples, can synthesize new images of the objects of interest. To match them to new images one seeks the model parameters, which minimize the difference between the target image and the synthesized image. The AAM essentially learns the relationship between the residual differences in such a match and the parameter displacements required to correct the current offset from the optimal position.

\section{RELATED WORK}

In recent years, there has been significant research efforts toward the development of automated methods for segmentation and cell tracking for living cells as in [1][2][3][4][5]. Most of the time, images from microscopic studies are corrupted during the recording process and due to the noise from the electronics devices, which affect the quality of the image.

Cell tracking with good accuracy is important in microscopic imaging studies. For instance, Image analysis of leukocytes cells is essential part for curative and preventative treatments to many diseases and also important to understand and successfully treat inflammatory diseases as in Ray et al. [23]. Sensitive tracking for moving cells is important to do mathematical modelling to cell locomotion. Zimmer [17] modified the Active Contour model to detect the mobility of the moving cells and also handle the cell division by providing an initial segmentation for the first frame. Mukherjee et al. [19] developed an algorithm by using threshold decomposition computed via image level sets to handle tracking problem and segmentation simultaneously. Li [20] developed an algorithm with two levels, a motion filter and a level set tracker to handle the cell detection and the cells that move in and out of the image. Coskun et al. [12] used imaging data to solve the inverse modelling problem to determine the mobility analysis of the cells. Recently there have been a number of researchers attempt to create automated algorithms to detect and track the cells from microscopic images as in Mélange [6]; and Mignotte [10].

This paper discusses the tracking cell accuracy as important task in many biological studies to understand the cell behaviour and the way in which cells interact with the world around them. One of the major goals of tracking the mobility of living cells is to find the best way to increase segmentation and tracking accuracy under weak image boundaries, over and under segmentation, which the most cell tracking challenge problems and responsible for lacking accuracy in cell tracking techniques. 


\section{ALGORITHM}

In this section, a comparison between tracking video sequences using AAM model and ASM model, to investigate the tracking quality between the two methods. In order to achieve that, a set of video sequences of 32 live cells previously collected and computed manual by Jung et al. (1998), is used to test the ability of the two models to track the video sequences of WBC frames, and making a comparison between the tracking quality of the two methods.

\subsection{Active Shape Model-Based Tracking (ASM)}

A shape consisting of $n$ points can be considered as one data point in $2 n$ dimensional space. A classical statistical method for dealing with redundancy in multivariate data is the principal component analysis (PCA). PCA determines the principal axes of a cloud of $n$ points at locations $x_{i}$. The principal axes, explaining the principal variation of the shapes, compose an orthonormal basis equation as $\Phi=\left\{P_{1}, P_{2}, \ldots, P_{n}\right\}$, of the covariance matrix:

$\sum=\frac{1}{n-1} \sum_{i-1}^{n}\left(x_{i}-\bar{x}\right)\left(x_{i}-\bar{x}\right)^{T}$. It can be shown that the variance across the axis corresponding to the $i^{\text {th }}$ eigenvalue $\lambda_{i}$ equals the eigenvalue itself. By deforming the mean shape $\bar{x}$, using a linear combination of eigenvectors $\Phi$, weighted by socalled modal deformation parameters $b$, and can generate an instance of the shape. Therefore, the new shape can be expressed in the following manner: $x=\bar{x}+\Phi b$. By varying the elements of $b$ and modify the shape. By applying constraints and ensure that the generated shape is similar to the mean shape from the original training data. Through applying limits of $\pm 3 \sqrt{\lambda_{i}}$ to each element $b_{i}$ of $b$, where $\lambda_{i}$ is the variance of the $i^{\text {th }}$ parameter $b_{i}$, and operate on plausible values of $b$. The deformation of the shape is constrained to a subspace spanned by a few eigenvectors corresponding to the largest eigenvalues. If all principal components are employed, ASM can represent any shape and no prior knowledge about the shape is utilized.

Shape Alignment: Given two 2D shapes, $x_{1}$ and $x_{2}$ our aim is to determine the parameters of a transformation $T$, which, when applied to $x_{2}$ can best align it with $x_{1}$ and one to one point correspondence. During alignment and utilize an alignment metric that is defined as the weighted sum of the squares of the distances between corresponding points on the considered shapes, and choose the parameters $t$ of the transformation $T$ to minimize $E$ :

$E=\sum_{i=1}^{n}\left(x_{1 i}-T_{t}\left(x_{2 i}\right)\right)^{T} W\left(x_{1 i}-T_{t}\left(x_{2 i}\right)\right)$

where $W$ is a diagonal matrix of weights $\left\{w_{1}, w_{2}, \ldots, w_{n}\right\}$. Expressing $T_{t}$ in the following form: 
$T_{t} \equiv\left[\begin{array}{ccc}s \cos (\theta) & -s \sin (\theta) & t_{x} \\ s \sin (\theta) & s \cos (\theta) & t_{y} \\ 0 & 0 & 1\end{array}\right]\left[\begin{array}{c}x_{2 i} \\ y_{2 i} \\ 1\end{array}\right]$

where denoting $a_{x}=s \cos (\theta), a_{y}=s \sin (\theta)$ and can rewrite equation (3.20) in the following form: $E=\sum_{i=1}^{n} w_{i}\left(\left(a_{x} x_{2 i}-a_{y} y_{2 i}+t_{x}-x_{1 i}\right)^{2}+\left(a_{y} x_{2 i}+a_{x} y_{2 i}-t_{y}-y_{2 i}\right)^{2}\right)$. The error $E$ assumes a minimal value when all the partial derivatives are zero. Differentiating the last equation with regard to $a_{x}$ is obtaining the following:

$\sum_{i=1}^{n} w_{i}\left(\left(a_{x}\left(x_{2 i}^{2}+y_{2 i}^{2}\right)+t_{x} x_{2 i}+t_{y} y_{2 i}\left(x_{1 i} x_{2 i}+y_{1 i} y_{2 i}\right)\right)=0\right.$.

Differentiating w.r.t. remaining parameters and equating to zero gives:

$$
\left[\begin{array}{l}
C_{1} \\
C_{2} \\
X_{1} \\
Y_{1}
\end{array}\right]=\left[\begin{array}{cccc}
D & 0 & X_{2} & Y_{2} \\
0 & D & -Y_{2} & X_{2} \\
X_{2} & -Y_{2} & W & 0 \\
Y_{2} & X_{2} & 0 & W
\end{array}\right]\left[\begin{array}{c}
t_{x} \\
t_{y} \\
a_{x} \\
a_{y}
\end{array}\right],
$$

where: $X_{k}=\sum_{i=1}^{n} w_{i} x_{k i}, Y_{k}=\sum_{i=1}^{n} w_{i} Y_{k i}, C_{1}=\sum_{i=1}^{n} w_{i}\left(x_{1 i} x_{2 i}+y_{1 i} y_{2 i}\right), C_{2}=\sum_{i=1}^{n} w_{i}\left(y_{1 i} x_{2 i}+x_{1 i} y_{2 i}\right)$, $D=\sum_{i=1}^{n} w_{i}\left(x_{2 i}^{2}+y_{2 i}^{2}\right), W=\sum_{i=1}^{n} w_{i}$. The parameters $t_{x}, t_{y}, a_{x}$ and $a_{y}$ constitute a solution which best aligns the shapes. An iterative approach to find the minimum of square distances between corresponding model and image points is as follows:

Step 1. Initialize shape parameter $b$ to zero.

Step 2. Generate the model instance $x=\bar{x}+\Phi b$.

Step 3. Find the pose parameters using equation (3.20), which best map $x$ to $Y$.

Step 4. Invert the pose parameters and then use to project image pixels $Y$ into the model coordinate frame: $y=T_{t}^{-1}(Y)$.

Step 5. Project $y$ into the tangent plane to $\bar{x}$ through scaling it by $1 /(y \cdot \bar{x}): y^{\prime}=y /(y \cdot \bar{x})$.

Step 6. Update $b$ to match $y^{\prime}$ as follows: $b=\Phi^{T}\left(y^{\prime}-\bar{x}\right)$.

Step 7. If not converged, repeat starting from step 2.

Step 8. Repeat all steps until all frames of the image sequence are processed. The result as a function of time enable estimation of the position and deformation of the corresponding cells for each frame in the sequence and update shape and position.

Step 9. Segmentation and tracking transforms the raw images into a file that encodes the boundaries of every cell at every time point, directly allowing computation of shape and motion 
parameters. These extracted data for shape and position can be used to quantify parameters characterizing cell shape and motion. Segmentation and tracking transforms the raw images into a file that encodes the boundaries of every cell at every time point, and allow the computation of shape and motion of the cells.

\subsection{Active Appearance Model-Based Tracking}

In this subsection, Cootes et al. (2002) introduces the Active Appearance Models (AAM) algorithm. The original algorithm was described by Edwards's et.al. (1998). The Active Appearance Model (AAM) algorithm has been widely used for matching statistical models of appearance to image data. The models, trained on suitably annotated examples, can synthesize new images of the objects of interest. To match them to new images one seeks the model parameters, which minimize the difference between the target image and the synthesized image. The AAM essentially learns the relationship between the residual differences in such a match and the parameter displacements required to correct the current offset from the optimal position.

Statistical Models of Appearance: An appearance model can represent both the shape and texture variability seen in a training set. The training set consists of labeled images, where key landmark points are marked on each example object. Using the notation of Cootes et.al. (1998), the shape of an object can be represented as a vector $x$ and the texture (grey-levels or colour values) represented as a vector $g$. The shape and texture are controlled by a statistical model of the form:

$$
\begin{aligned}
& x=\bar{x}+P_{s} b_{s} \\
& g=\bar{g}+P_{g} b_{g}
\end{aligned}
$$

where $b_{s}$ are shape parameters, $b_{g}$ are texture parameters. Since often shape and texture are correlated and can take this into account in a combined statistical model of the form:

$$
\begin{aligned}
& b_{s}=C_{s} c \\
& b_{g}=C_{g} c
\end{aligned}
$$

The combined appearance model has parameters, $c$, controlling the shape and texture at the same time. Combining the equations $(3.22,3.23)$ and $(3.24,3.25)$ gives:

$$
\begin{aligned}
& x=\bar{x}+Q_{s} c \\
& g=\bar{g}+Q_{s} c
\end{aligned}
$$

where $\bar{x}$ is the mean shape (in a normalized frame), $\bar{g}$ the mean texture and $Q_{s}, Q_{g}$ are matrices describing the modes of variation derived from the training set. To generate the positions of points in an image and use the following equation: 
$X=T_{t}(x)$

where $x$ are the points in the model frame, $X$ are the points in the image, and $T_{t}(x)$ applies a global transformation with parameters $t$. For instance, in $2 \mathrm{D}, T_{t}(x)$ is commonly a similarity transform with four parameters describing the translation, rotation and scale. The texture in the image frame is generated by applying a scaling and offset to the intensities, $g_{\text {im }}=T_{u}(g)$ where $u$ is the vector of transformation parameters. AAM Tracking: The AAM algorithm is a method of rapidly matching an appearance model to an image. It is a form of gradient descent algorithm, in which the gradient is assumed to be fixed at all iterations, and can thus be estimated in advance from a training set. This allows efficient matching to take place, even when there are many model parameters. The original matching algorithm was first described by Edwards's et.al. (1998) and expanded and refined by Cootes et.al. (2001). Basic algorithm: The appearance model parameters, $c$, and shape transformation parameters, $t$, define the position of the model points in the image frame, $X$, which gives the shape of the image patch to be represented by the model. During matching the pixels in this region of the image, $g_{\text {im }}$, are sampled and projected into the texture model frame, $g_{s}=T^{-1}\left(g_{i m}\right)$. The current model texture is given by $g_{m}=g+Q_{g} c$. The difference between model and image (measured in the normalized texture frame) is giving by the equation (3.30):

$r(P)=g_{s}-g_{m}$

where $P$ are the parameters of the model, $P^{T}=\left(c^{T}\left|t^{T}\right| u^{T}\right)$. A simple scalar measure of difference is the sum of squares of elements of $r, E(P)=r^{T} r$. In addition, Cootes et al., (2001) can be used to minimize and modify the parameters the following:

$\delta P=-R r(P) \quad$ where, $\quad R=\left(\frac{\partial r^{T}}{\partial P} \frac{\partial r}{\partial P}\right)^{-1} \frac{\partial r^{T}}{\partial P}$

$\frac{\partial r}{\partial P}$ is estimated by numeric differentiation, systematically displacing each parameter from the known optimal value on typical images and computing an average over the training set. $R$ is computed and used in all subsequent searches with the model.

Basic AAM Search: Equation (3.30) can be used to suggest a correction to make in the model parameters based on a measured residual $r$. Given a current estimate of the model parameters, $c$, the pose $t$, the texture transformation $u$, and the image sample at the current estimate, $g_{\text {im }}$, one step of the iterative matching procedure is as follows:

step1. Project the texture sample into the texture model frame using $g_{s}=T^{-1}\left(g_{i m}\right)$

step 2. evaluate the error vector, $r=g_{s}-g_{m}$, and the current error, $E=|r|^{2}$

step 3. compute the predicted displacements, $\delta P=-R r(P)$ 
step 4. update the model parameters $P \rightarrow P+k \delta P$, where initially $k=1$,

step 5. calculate the new points, $X^{`}$ and model frame texture $g_{m}$,

step 6. sample the image at the new points to obtain $g_{\text {im }}$

step 7. calculate a new error vector, $r^{\prime}=T_{u}^{-1}\left(g_{i m}^{\prime}\right)-g_{m}^{\prime}$

step 8. if $|r|^{2} \leq E$ then accept the new estimate, otherwise try at $k=0.5, k=0.25$ etc.

This procedure is repeated until no improvement is made to the error, $\left|r^{\prime}\right|^{2}$, and convergence is declared (or a maximum number of iterations is reached).

Shape AAM: Cootes et.al. (1998), proposed a variant on the AAM in which instead of the residuals driving the appearance model parameters, $c$, they could be used to drive the pose, $t$, and shape model parameters, $b_{s}$, alone. The texture model parameters could then be directly estimated by fitting to the current texture, and $d t=R_{t} r, d b_{s}=R_{s} r$. The matching procedure modified as follows:

step 1. Normalize the current texture sample to obtain $g_{s}$.

step 2. Fit the texture model to the sample using $b_{g}=P_{g}^{T}\left(g_{s}-\bar{g}\right)$.

step 3. Compute the residual as $r=g_{s}-g_{m}$.

step 4. Predict the pose and shape parameter updates using Edwards et al. (1998).

step 5. Apply and test the updates as for the basic algorithm.

Step 6. Repeat all steps until all frames of the image sequence are processed. The result as a function of time enable estimation of the position and deformation of the corresponding cells for each frame in the sequence and update shape and position.

Step7. Segmentation and tracking transforms the raw images into a file that encodes the boundaries of every cell at every time point, directly allowing computation of shape and motion parameters. These extracted data for shape and position can be used to quantify parameters characterizing cell shape and motion. Segmentation and tracking transforms the raw images into a file that encodes the boundaries of every cell at every time point, and allow the computation of shape and motion of the cells.

These extracted data for shape and position can be used to quantify parameters characterizing cell shape and motion. Segmentation and tracking transforms the raw images into a file that encodes the boundaries of every cell at every time point, and allow the computation of shape and motion of the cells.

\subsection{Accuracy Performance Measure}

The performance of the enhanced cell tracking technique is measured by how well the system can track the WBC. Two methods are used to evaluate the enhanced technique. 
- Percentage of frames tracked. If a computed cell center is within one cell radius of the manually observed cell center, then we consider that frame as tracked. The percentage is computed as the ratio of number of frames tracked to the total number of frames in the sequence.

$$
f_{P}=\frac{N}{N_{\text {total }}}
$$

where $f_{P}$ is the percentage of frames tracked, $N$ is the number of frames tracked, and $N_{\text {total }}$ is the total number of frames in the image sequence.

- The second method is used to measure the performance of our enhanced technique is calculating the Root Mean Squared Error (RMSE) between the manual (ground truth) and the computed displacement. In addition, compare the RMSE achieved with the RMSE for the other methods (Jung et al., (1998), and also earlier observation of Ley et al., (1996)).

The RMSE (in microns) describes how accurately the tracker tracks the cell as compared predicted (computed) to the actual (ground truth or manual) data. RMSE gives the standard deviation of the model prediction error.

A smaller value indicates better model performance. The root mean square error (RMSE) is giving a sense of the predicted values error. Also how close the predicted values are to the actual values. The RMSE mathematical formula is giving by:

$R M S E=\sqrt{\frac{\sum_{i=1}^{n}\left(X_{\text {actual }, i}-X_{\text {predicted }, i}\right)^{2}}{n}}$

where $X_{\text {actual }}$ is actual values and $X_{\text {predicted }}$ is predicted values, and $i$ represent the current predictor, and $n$ represents the number of predictors. The combination of the percentage of frames tracked and the RMSE yields the qualitative performance Ratings.

\subsection{Validation and Benchmarks}

To validation and evaluate the quality of an automated cell tracking procedure, a comparison have been made between the manually marked data, also known as ground-truth, of three video sequences protocols and the automated extracted data, to compare a computationally produced tracking with the ground truth annotation.

In addition, compare the experiment results with the other research methods results such as Jung et al., (1998), Scott et al. (2001), and earlier observation of Ley et al. (1996).

\section{RESULTS AND DISCUSSION}


An Active Appearance Model (AAM) is a statistical model that describes both the shape of an object and the image texture around the shape. The AAM is an extension of an Active Shape Model (ASM).

As shown in Table 4.4, Figure 4.11, and Figure 4.12, the result of the comparison between the AAM, ASM automated methods, and the Manual calculation shows that AAM has better accuracy in tracking the WBC, where the results of AAM is similar to the manual calculation(ground truth).

The main objective here is comparing the mobility results between the two methods. The results indicate the ability of AAM to give better accuracy for tracking the mobility of the WBC.

The conclusion for the comparison shows that although an AAM may be more robust than an ASM, but an ASM tends to have a larger capture range if started from a poorly initialized solution. This is because an ASM searches around the current location, whereas the AAM only examines the image directly under its current area.

Table 4.1: Some mobility results for both Manual(ground truth), (AAM) and (ASM) tracking of the leukocytes cells.

\begin{tabular}{|c|c|c|c|c|c|c|}
\hline Cell & $\begin{array}{l}\mathrm{Vel}_{G T} \\
(\mu \mathrm{m} / \mathrm{s}\end{array}$ & $\begin{array}{l}\text { Vel } \\
\text { (Experimen } \\
\text { tal) }(\mu \mathrm{m} / \mathrm{s})\end{array}$ & $\begin{array}{l}\text { Vel } \\
\text { ASM } \\
\text { (Experimen } \\
\text { tal) } \\
(\mu \mathrm{m} / \mathrm{s})\end{array}$ & ShapeIndex $_{G T}$ & $\begin{array}{l}\text { Shape.Index } x_{A A M} \\
\text { (Experimental) }\end{array}$ & $\begin{array}{l}\text { Shapeindex } x_{A S M} \\
\text { (Experiment) }\end{array}$ \\
\hline 1 & 7.0 & 7.2 & 6.7 & 2.4 & 2.2 & 2.3 \\
\hline 2 & 3.5 & 3.4 & 3.1 & 2.7 & 2.7 & 2.5 \\
\hline 3 & 2.5 & 2.4 & 2.6 & 1.5 & 1.7 & 1.4 \\
\hline 4 & 2.1 & 2.2 & 2.4 & 2.2 & 2.3 & 2.3 \\
\hline 5 & 3.1 & 3.3 & 3.2 & 2.5 & 2.6 & 2.1 \\
\hline 6 & 5.6 & 5.7 & 5.8 & 1.5 & 1.5 & 1.4 \\
\hline 7 & 6.8 & 6.7 & 6.3 & 1.3 & 1.2 & 1.5 \\
\hline 8 & 7.5 & 7.5 & 7.2 & 2.5 & 2.6 & 2.4 \\
\hline 9 & 1.3 & 1.2 & 3.3 & 2.9 & 2.9 & 2.5 \\
\hline 10 & 2.4 & 2.4 & 2.1 & 1.7 & 1.8 & 1.6 \\
\hline 11 & 3.5 & 3.6 & 3.2 & 2.5 & 2.6 & 2.2 \\
\hline 12 & 7.6 & 7.6 & 7.1 & 2.6 & 2.4 & 2.4 \\
\hline 13 & 2.8 & 2.7 & 2.4 & 2.8 & 2.7 & 2.5 \\
\hline 14 & 2.6 & 2.8 & 2.1 & 2.1 & 2.2 & 1.9 \\
\hline 15 & 2.4 & 2.4 & 2.2 & 1.2 & 1.3 & 1.4 \\
\hline 16 & 7.5 & 7.4 & 7.2 & 2.6 & 2.5 & 2.2 \\
\hline 17 & 4.3 & 4.4 & 4.1 & 2.3 & 2.1 & 1.9 \\
\hline 18 & 4.5 & 4.4 & 4.3 & 1.3 & 1.4 & 1.8 \\
\hline 19 & 7.8 & 7.7 & 7.3 & 2.5 & 2.4 & 2.1 \\
\hline 20 & 1.6 & 1.7 & 1.5 & 1.1 & 1.2 & 1.5 \\
\hline
\end{tabular}


International Journal of Information Technology Convergence and Services (IJITCS) Vol.6, No.4, August 2016

$\mathrm{Vel}_{G T}$ :GROUND TRUTH $\quad \operatorname{Vel}_{\text {AAM }}:$ AAM VELOCITY (EXPERIMENTAL) $\quad \operatorname{Vel}_{\text {ASM }}:$ ASM

VELOCITY (EXPERIMENTAL)

Shape.Index $_{G T}$ :GROUND TRUTH (CHANGE SHAPE INDICATOR) Shape.index ${ }_{A A M}$ : CHANGE SHAPE

(EXPERIMENTA) Shape.index ${ }_{A S M}$ :CHANGE SHAPE(EXPERIMENTAL)

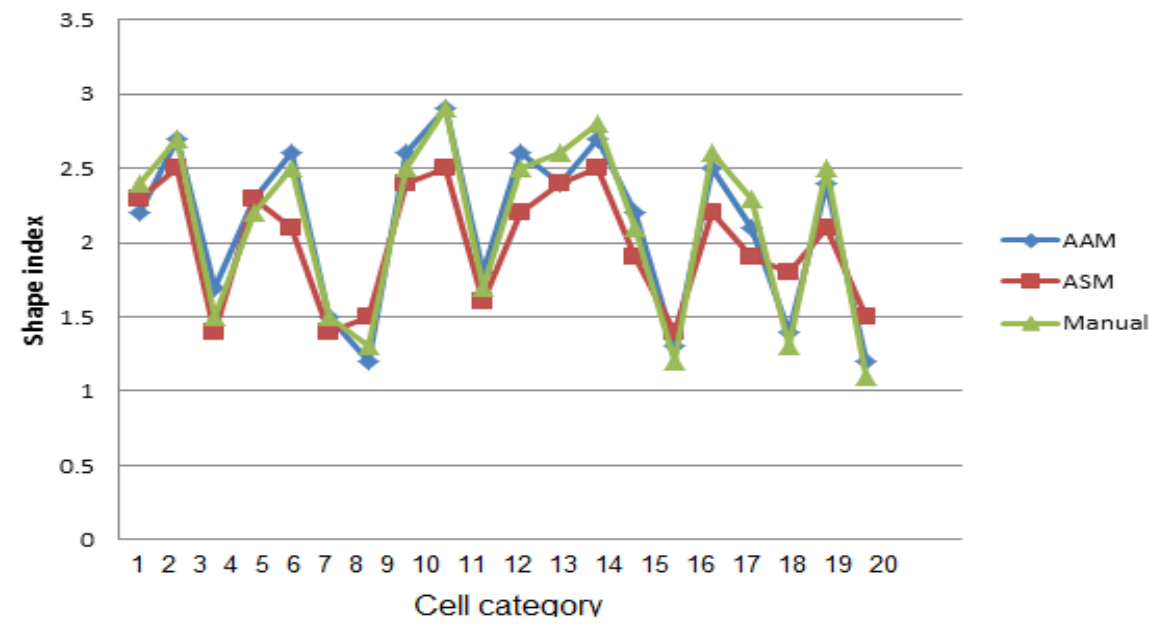

Figure 4.1: Experiments results for the automated deformation tracking results for the tracked cells from video sequences. A comparison between AAM, ASM, and the manual calculation from Jung et al. (1998).

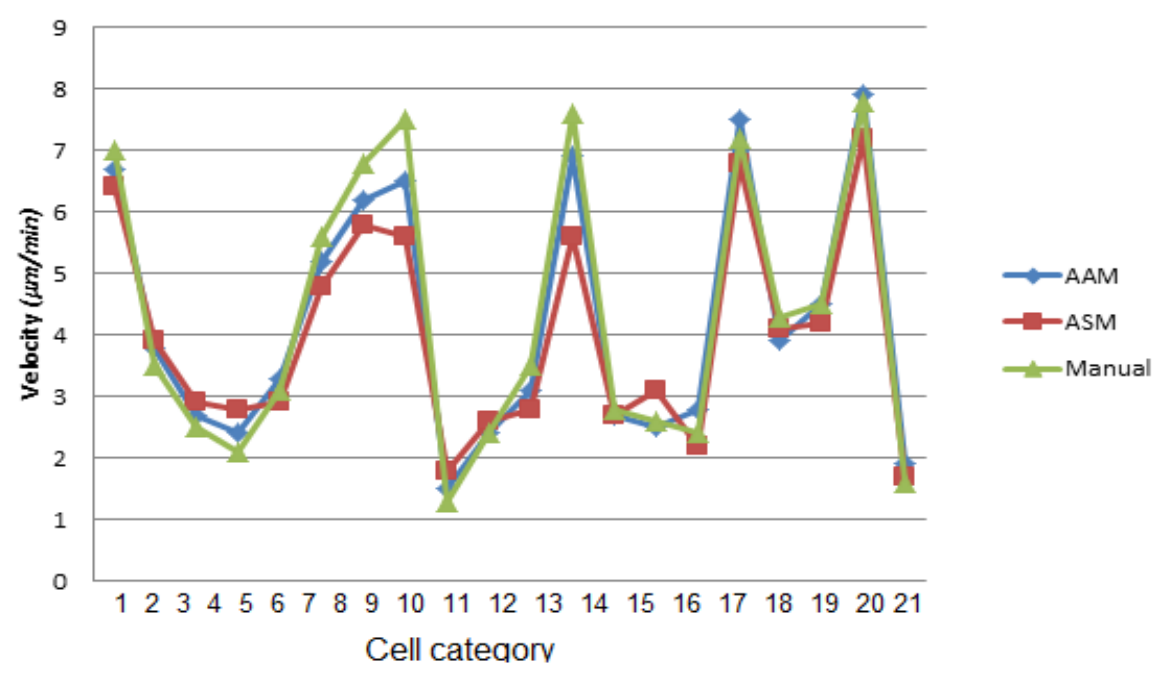

Figure 4.2: Experiments results for the automated velocity tracking results for the tracked cells from video sequences. A comparison between AAM, ASM, and the manual calculation from Jung et al. (1998). 


\section{CONCLUSION}

In this paper, a comparison between cell tracking using active shape model (ASM) and active appearance model (AAM) algorithms has been made, to investigate the cells tracking quality between the two methods. Sensitive and accurate cell tracking system is essential to cell motility studies. The active shape model (ASM) and active appearance model (AAM) algorithms has proved to be a successful methods for matching statistical models.

The comparison between (AAM), (ASM) methods, and the manual calculation (ground truth) method based on experimental result, shows that the (AAM) algorithm results indicate the ability of (AAM) method to give better accuracy for tracking the mobility of the WBC than (ASM) method.

\section{REFERENCES}

[1] Aly, Ashraf A., Deris, S., Zaki, N., (2014) "Intelligent Algorithms for Cell Tracking and Image Segmentation", International Journal of Computer Science \& Information Technology (IJCSIT) Vol 6, No 5, October 2014.

[2] Jie, S., Hao, F., Guoping, Q., Philip, K., and Mohammad, I. (2013) "Segmenting Overlapping Cell Nuclei In Digital Histopathology Images", 35th Annual International Conference of the IEEE (EMBS Osaka).

[3] Siegel, R., Naishadham, D., Ahmedin, J. (2013) "Cancer Statistics", 2013\|, CA Cancer J Clin, 63:11- 30 .

[4] Anders, E., Dufort, P., Daniel, F., and Stephen, L. (2013) "Medical Image Processing on the GPU: Past, Present and Future" Medical Image Analysis.

[5] Xin, Q., Fuyong, X., David, Foran J., and Lin Y. (2012) "Robust Segmentation of Overlapping Cells in Histopathology Specimens Using Parallel Seed Detection and Repulsive Level Set" IEEE Trans. On Biomed. Eng., vol. 59, no. 3.

[6] Aly, Ashraf A., Deris, S., Zaki, N., (2011) "A Novel Image Segmentation Enhancement Technique Based On Active Contour and Topological Alignments ", Advanced Computing: An International Journal (ACIJ), Vol.2, No.3, May 2011.

[7] Danuser, G., Meijering, E., and Smal, I. 2011. Tracking in molecular bioimaging. Signal Processing Magazine IEEE, 23(3):46-53.

[8] Melange, T., Nachtegael, M., and Kerre, E. 2011. Fuzzy Random Impulse Noise Removal From Color Image Sequences. IEEE Trans. 20: 1023 - 1035.

[9] Danuser, G., Meijering, E., and Smal, I. 2011. Tracking in molecular bioimaging. Signal Processing Magazine, IEEE, 23(3):46-53.

[10] Mignotte, M. 2010. A Label Field Fusion Bayesian Model and Its Penalized Maximum Rand Estimator for Image Segmentation. IEEE Trans. 19: 1610 - 1624.

[11] Bradski, G. 2000. The Open CV Library. Dr. Dobb's Software Tools for the Professional Programmer.

[12] Coskun, H., Li, Y., and Mackey, M. A. 2007. Ameboid cell motility: A model and inverse problem, with an application to live cell imaging data. Journal of heoretical Biology, 244(2):169-179.

[13] Kass, M., Witkin, A., and Terzopoulos, D. 1987. Snakes: Active Contour models. International Journal of Computer Vision, pages 321-331.

[14] Li, K., Miller, E., Weiss, L., Campbell, P., and Kanade, T. 2006. Online tracking of migrating and proliferating cells imaged with phase - contrast microscopy", Proc. of the 2006 Conf, on Computer Vision and Pattern Recognition Workshop (CVPRW'06), pages 65-72.

[15] Mukherjee, D., Ray, N., and Acton, S., 2004. Level set analysis for leukocyte detection and tracking. IEEE Trans Image Process, 13(4)562-72. 
[16] Ray, N., Acton, S., and Ley, K. 2002. Tracking leukocytes in vivo with shape and size constrained Active Contours, IEEE Trans Med Imaging 21(10):1222-1235.

[17] Zimmer, C., Zhang, B., Dufour, A., Thebaud, S., Berlemont, V. and Marin, O. 2006. On the Digital Trail of Mobile Cells. Signal Processing Magazine, 23(3):54-62.

[18] Miura, K., 2005. Tracking Movement in Cell Biology. Advances in Biochemical Engineering Biotechnology, 95:267-295.

[19] Mukherjee, D., Ray, N., and Acton, S. 2004. Level set analysis for leukocyte detection and tracking. IEEE Trans Image Process, 13(4):562-572.

[20] Li,Y., Zheng,Y., Doermann, D., and Jaeger, S. 2008. Script-Independent Text Line Segmentation Segmentation in Freestyle Handwritten Documents. IEEE Trans Pattern Anal Mach Intell, 30(8):1313-1329.

[21] Ersoy, I., Bunyak, F., Mackey, M., and Palaniappan, K. 2008. Cell Segmentation Using HessianBased Detection and Contour Evolution with Directional Derivatives. International Conference on Image Processing, 1804-1807.

[22] Jung, U., Norman, K., Ramos, C., Scharffetter-Kochanek, K., Beaudet, A., and Ley, K. 1998. Transit time of leukocytes rolling through venules controls cytokine- induced inflammatory cell recruitment in vivo. J. Clin. Invest. 102, 1526-1533.

[23] Ray, N., Acton, S., and Ley, K. 2002. Tracking leukocytes in vivo with shape and size constrained Active Contours", IEEE Trans Med Imaging, 21(10):1222-1235.

[24] Debeir, O., Ham, P., Kiss, R., and Decaestecker C. 2005. Tracking of migrating cells under phase-contrast video microscopy with combined mean-shift processes. IEEE Trans Med Imaging , 24(6):697-711.

[25] Sacan, A., Ferhatosmanoglu, H., and Coskun, H. 2008. CellTrack: an opensource software for cell tracking and motility analysis. Bioinformatics, 24(14):1647-1649.

[26] Rodrigo, M., and Leyza, B. 2007. White blood cell segmentation using morphological operators and scale - space analysis. Brazilian Symposiun on computer graphics and Image Processing, 1530-1834/07.

[27] Scott, T., Klaus, W., and Klaus, L. 2002. Automatic Tracking of Rolling Leukocytes in Vivo, Microvascular Research, vol 63, pp. 139 - 148.

\section{AUTHOR}

Dr. ASHRAF ALY

Morehead state University

Morehead, KY 40351, USA

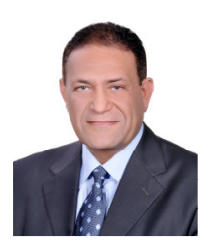

